\title{
Susceptibilidad de Biomphalaria tenagophila de las cuencas de los ríos Paraná y Uruguay a Schistosoma mansoni ${ }^{1}$
}

\author{
C. Edgardo Borda ${ }^{1}$ y María Josefa Felisa Rea ${ }^{2}$
}

RESUMEN Con el fin de estudiar la posibilidad de extensión de la esquistosomiasis a las cuencas de los ríos Paraná y Uruguay se expusieron experimentalmente a infección por Schistosoma mansoni 1711 caracoles criados en laboratorio a partir de ejemplares de Biomphalaria tenagophila recolectados en 15 poblaciones de dicha zona geográfica. Se utilizaron tres cepas de S. mansoni: la BH2, adaptada a B. glabrata, y las cepas SJ y SJ2, adaptadas a B. tenagophila. No se produjo infección de ninguno de los 543 caracoles expuestos a la cepa BH2 ni de los 668 expuestos a la SJ. En cambio, de los 500 expuestos a la cepa SJ2, fueron susceptibles a la infestación 4 de 163 caracoles (2\%) procedentes de Ayolas, una localidad de la cuenca del Paraná, y 8 de 45 (18\%) de los procedentes de Fuente Salto, en la cuenca del Uruguay. Estos hallazgos son similares a los de otras áreas geográficas en las que se han encontrado poblaciones de B. tenagophila que no se infectan y otras que sí son susceptibles a S. mansoni. Los resultados de este trabajo señalan la posibilidad de expansión de la esquistosomiasis a una amplia región de Sudamérica en la que se halla B. tenagophila.

De las cinco especies de esquistosomas que afectan al hombre, únicamente Schistosoma mansoni es endémico en Sudamérica, siendo los países afectados el Brasil, Suriname y Venezuela (1). En el Brasil existen focos endémicos en los estados sureños de Paraná y Santa Catarina, limítrofes con la Argentina y el Paraguay (2). Sin embargo, en Argentina, Uruguay y Paraguay las encuestas epidemiológicas realizadas hasta 1985 no han

\footnotetext{
1 Trabajo realizado con apoyo económico de la Secretaría General de Ciencia y Técnica de la Universidad Nacional del Nordeste, Corrientes, Argentina.

2 Centro Nacional de Parasitología y Enfermedades Tropicales, Facultad de Medicina, Universidad Nacional del Nordeste, Santa Fe 1432, 3400 Corrientes, Argentina. Telefax 054-783-25484; Internet: cenpetrop@fmunne.ld.ar.
}

demostrado transmisión y tampoco se han notificado casos humanos recientes (3-7).

Las cuencas de los ríos Paraná y Uruguay, en territorios fronterizos de Paraguay, Argentina, Brasil y Uruguay (figura 1), no solo tienen un clima favorable para la transmisión de la esquistosomiasis sino también condiciones socioeconómicas apropiadas: migraciones sin control sanitario a través de la frontera, desde y hacia las áreas endémicas del Brasil, y poblaciones de bajo nivel socioeconómico asentadas en localidades con saneamiento e infraestructura sanitaria deficientes. Por ahora, de las condiciones biológicas importantes para la transmisión faltan los casos humanos, pero se han detectado transmisores potenciales, tal como muestra el presente estudio.
Por las razones mencionadas iniciamos estudios básicos relacionados con la posible propagación de la esquistosomiasis a las cuencas de los ríos Paraná y Uruguay. Estos trabajos, que pretenden ser parte de una adecuada vigilancia epidemiológica, se iniciaron en enero de 1993. Su objetivo específico es determinar la distribución geográfica de especies de caracoles Biomphalaria potencialmente intermediarias para la esquistosomiasis, así como la susceptibilidad de esas especies a diferentes cepas de S. mansoni procedentes del Brasil. Cada trimestre se realiza la búsqueda, colecta, identificación y cría de estos caracoles planórbidos. Se sabe que en las cuencas de los ríos Paraná y Uruguay, tanto en la Argentina (Misiones, Corrientes, Chaco y Entre Ríos), como en el Paraguay (Itapúa y Las 
FIGURA 1. Mapa de la región de estudio, que indica las localidades donde se recolectaron ejemplares de Biomphalaria tenagophila en los que se estudió la susceptibilidad a esquistosoma (• representa poblaciones de B. tenagophila no susceptibles; ${ }^{*}$ representa poblaciones susceptibles)

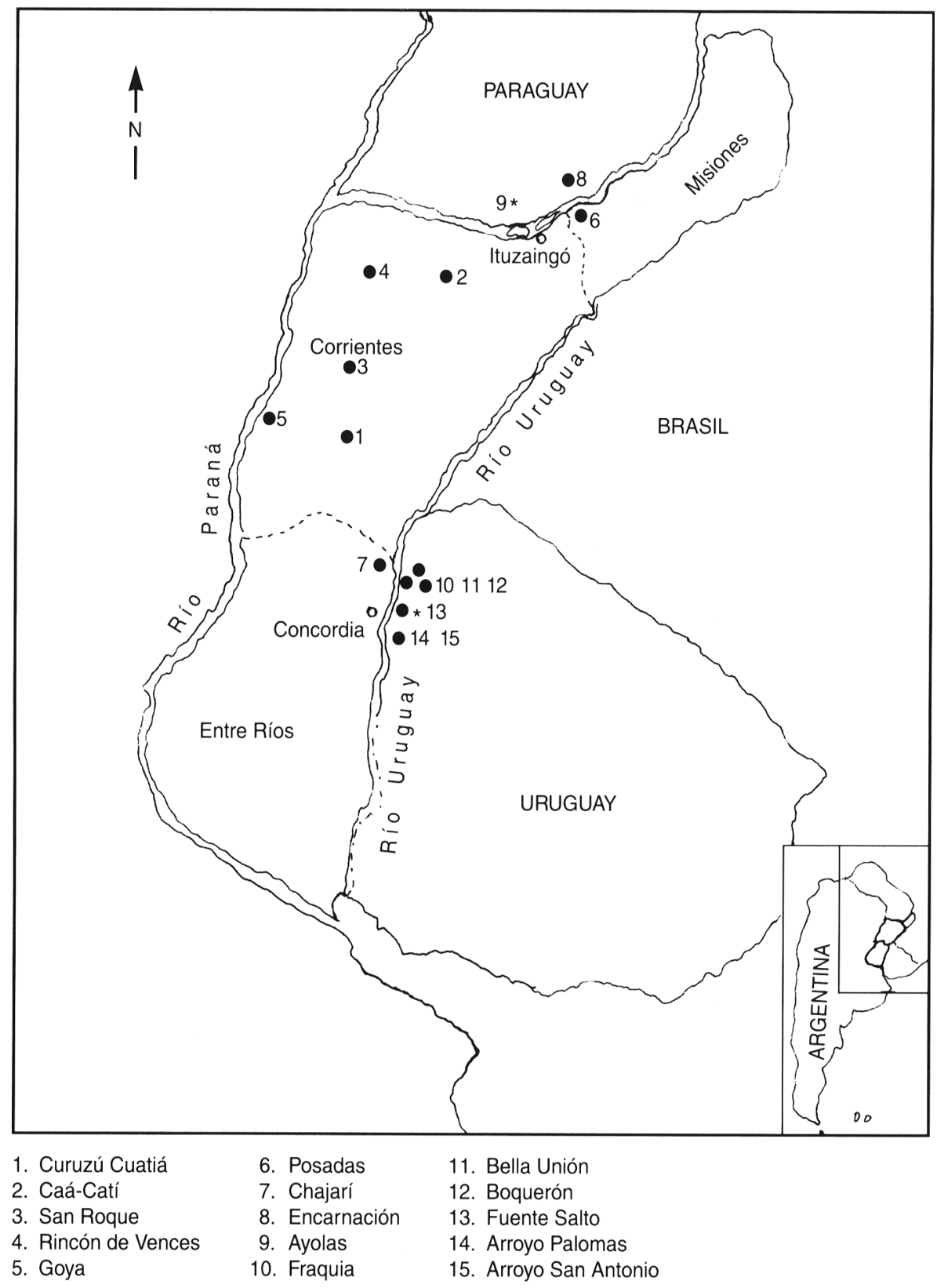

Misiones) y en el Uruguay (Artigas y Salto) existen poblaciones de $B$. tenagophila, B. straminea y B. peregrina (8).

En el Brasil los transmisores naturales son B. glabrata, B. straminea y B. tenagophila. En la región que hemos investigado no encontramos B. glabrata. Nuestra intención es estudiar la

\section{MATERIAL Y MÉTODOS}

En este trabajo se utilizaron ejemplares de B. tenagophila Orbigny, año 1835. Los caracoles fueron capturados en las cercanías de 15 poblaciones de Argentina, Paraguay y Uruguay (cuadro 1 y figura 1). Los moluscos progenitores recolectados en el campo y ambientados en el laboratorio fueron sacrificados según la técnica de Deslandes (9). Las claves sistemáticas para la identificación fueron las de Paraense (10).

De cada hábitat natural se recogieron como mínimo 10 ejemplares de diferentes diámetros que fueron transportados en condiciones adecuadas hasta el laboratorio (Centro Nacional de Parasitología y Enfermedades Tropicales, Corrientes, Argentina). Fueron colocados en acuarios y examinados una vez por semana durante un mes para determinar si estaban infectados con S. mansoni. Para ello cada caracol fue colocado en un recipiente apropiado con agua desclorada y expuesto durante una hora a luz artificial, para favorecer la emergencia de las cercarias. A continuación se procedió a su examen mediante microscopio estereoscópico. No se detectó infección natural en ninguno de los caracoles colectados.

Los caracoles fueron criados en condiciones experimentales, utilizando tierra extraída de su hábitat natural como sustrato de los acuarios y lechuga como alimento. Cuando comenzaron la ovipostura, su tamaño (unos $10 \mathrm{~mm}$ ) ya permitía la disección anatómica. Entonces se escogieron 10 ejemplares de cada hábitat y se procedió a su identificación.

Los caracoles B. tenagophila fueron expuestos experimentalmente a las cepas SJ y SJ2 de S. mansoni, adaptadas a $B$. tenagophila, y a la cepa $\mathrm{BH} 2$, adaptada a B. glabrata. Las tres cepas fueron aisladas por Paraense y Correa $(11,12)$. La cepa SJ se obtuvo de caracoles $B$. tenagophila naturalmente infectados en su hábitat natural en São José dos Campos, São Paulo, Brasil. Sin embargo, debido a discontinuidad en el mantenimiento de esta cepa hubo que recurrir 
a la SJ2, aislada en 1982 a partir de B. tenagophila infectada en el mismo biótopo de São José dos Campos, São Paulo. La cepa BH2 se obtuvo en 1985 de ejemplares de B. glabrata capturados en una acequia ubicada en Ressaca, cerca de la ciudad brasileña de Belo Horizonte, Minas Gerais. Las cepas de S. mansoni se mantienen en nuestro laboratorio mediante pasajes a través de caracoles simpátridas, hámster Mesocricetus auratus y ratón Mus musculus variedad albina.

Según Files (13), una vez expuesto a los miracidios de S. mansoni, un caracol se considera infectado cuando produce o contiene cercarias. La infectividad de estas se verificó con el ratón albino. Se calculó un índice porcentual de caracoles infectados correspondiente a cada localidad respecto al total de caracoles de esa procedencia expuestos a miracidios de $S$. mansoni.

Los miracidios de S. mansoni fueron obtenidos a partir de huevos retenidos en el hígado e intestino de ratones albinos y cricetos (hámsteres) experimentalmente infectados con 60 y 100 cercarias, respectivamente y sometidos a autopsia 7 semanas después de la exposición. La suspensión de esos tejidos homogeneizados en solución salina al 0,85\% se dejó sedimentar en la oscuridad a temperatura de refrigerador (aproximadamente $4^{\circ} \mathrm{C}$ ). Después de varios lavados por decantaciones sucesivas, el sedimento fue suspendido en agua desclorada. La eclosión de los miracidios se estimuló mediante exposición a luz de lámpara eléctrica. Se reguló la distancia de los focos luminosos de tal modo que la temperatura del agua fuera de unos $28^{\circ} \mathrm{C}$, lo que favorece considerablemente la eclosión (14). Se concentraron los miracidios tal como propone Chaia (15) y su número fue determinado según la técnica de Paraense y Correa (16). Para la exposición en masa, una vez comprobada la presencia de miracidios en la suspensión de agua desclorada, esta fue vertida en frascos que contenían 50 caracoles en $1500 \mathrm{~mL}$ de agua, colocados en acuarios mayores con agua a temperatura constante $\left(28^{\circ} \mathrm{C}\right)$.

CUADRO 1. Susceptibilidad de caracoles Biomphalaria tenagophila de 15 localidades de las cuencas de los ríos Paraná y Uruguay a infección con cepas de Schistosoma mansoni

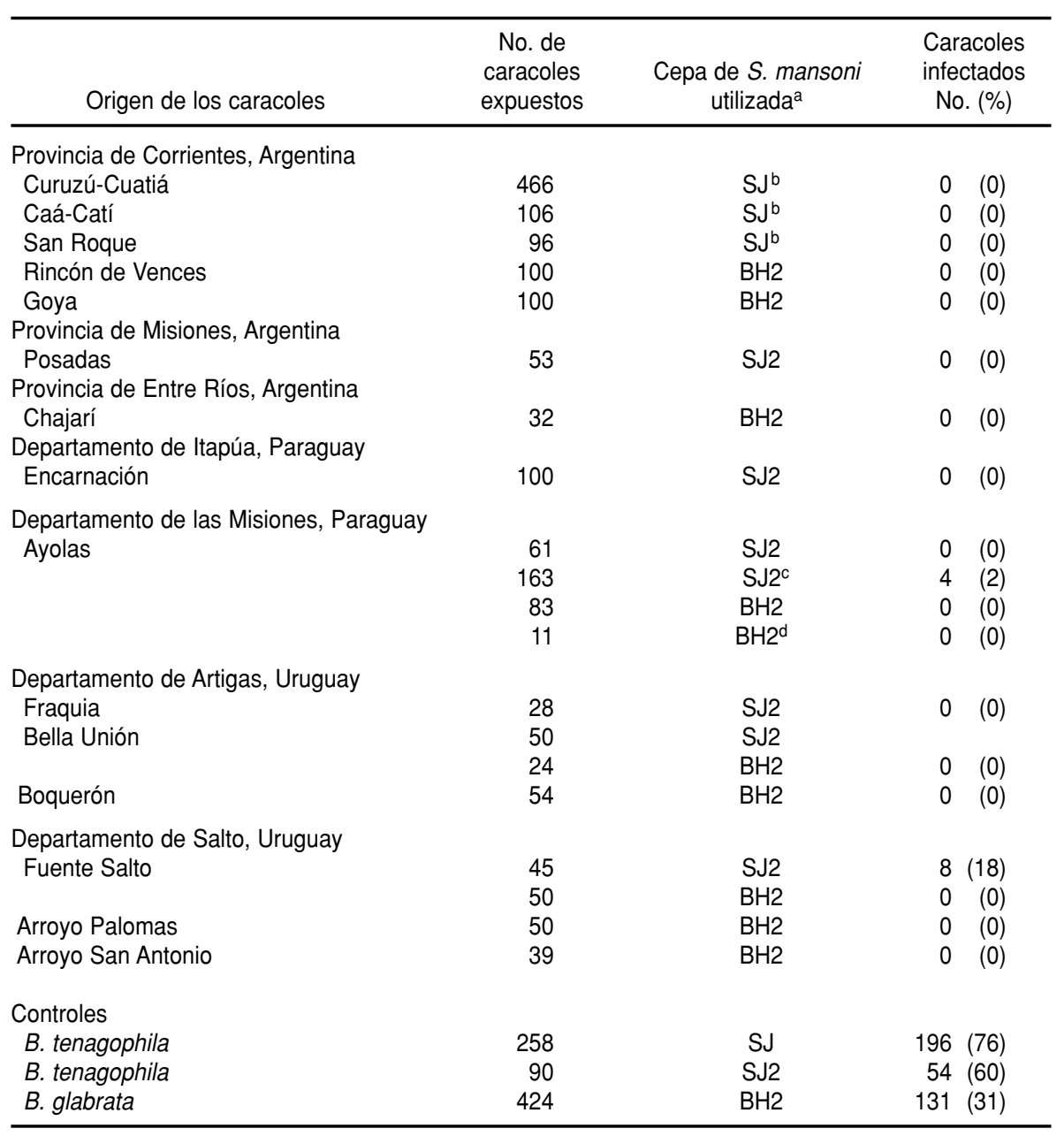

a Cada ejemplar de $B$. tenagophila fue expuesto a 10 miracidios de $S$. mansoni, salvo los casos en los que se indica otra cosa. ${ }^{b}$ Los ejemplares de $B$. tenagophila fueron expuestos a miracidios en masa.

${ }^{c}$ Cada ejemplar de $B$. tenagophila fue expuesto a 50 miracidios.

d Cada ejemplar de $B$. tenagophila fue expuesto a 100 miracidios.

Tanto en la exposición individual como en masa, los ejemplares de B. tenagophila fueron llevados a otros acuarios de 8 a 12 horas después del baño de exposición.

Exceptuando los ensayos en que se expusieron los caracoles en masa y en forma individual a 50 y 100 miracidios, se usaron 10 miracidios por cada caracol para que los resultados fueran comparables. En general, el diámetro de la concha de los moluscos varió de 4 a $8 \mathrm{~mm}$. Entre los 28 y 30 días posteriores a la exposición y después cada 5 días, se colocaron los caracoles individualmente en frascos que contenían $3 \mathrm{~mL}$ de agua y que se colocaban bajo la luz de lámparas eléctricas para inducir la emergencia de cercarias. Una vez transcurridos 90 días desde la exposición sin que hubieran aparecido cercarias, los caracoles se sacrificaron entre placas de vidrio y fueron examinados al microscopio estereoscópico. Esta técnica fue utilizada también para examinar los ejemplares muertos durante el período de observación. Las cercarias eliminadas por los ejemplares de $B$. te- 
nagophila de Ayolas y Fuente Salto fueron recolectadas y a ellas fueron expuestos dos lotes de ratones albinos, cada lote de 10 ratones. Cada ratón fue expuesto a 60 cercarias. A partir de los 35 días, se comprobó la infección observando los huevos eliminados con las heces de cada ratón, de acuerdo con la técnica de Borda y Pellegrino (17). Los ratones fueron sacrificados a los 50 días para retirar los esquistosomas adultos del sistema venoso, mediante la técnica de perfusión descrita por Pellegrino y Siqueira (18). Para la perfusión del hígado se utilizó solución fisiológica y para la del mesenterio, goma arábica a $4 \%$ en solución fisiológica. Los esquistosomas se recolectaron en forma separada en un recipiente esmaltado, para conocer su distribución y número. Como controles del índice de infección se utilizaron especies simpátridas de Biomphalaria que se expusieron a las cepas de $S$. mansoni. Para la BH2, 424 B. glabrata de 8 a $10 \mathrm{~mm}$ de diámetro y para la SJ y la SJ2, 258 y 90 B. tenagophila, respectivamente, de 4 a $8 \mathrm{~mm}$ de diámetro. Cada caracol control fue expuesto individualmente a 10 miracidios de la cepa correspondiente.

\section{RESULTADOS}

Fueron expuestos a miracidios de S. mansoni un total de 1711 ejemplares de B. tenagophila: 668 a la cepa SJ, 500 a la SJ2 y 543 a la BH2. Únicamente los caracoles de Ayolas, Bella Unión y Fuente Salto fueron expuestos a las cepas adaptadas a B. tenagophila (SJ2) y B. glabrata (BH2) (cuadro 1). Esto se debió a la discontinuidad en el mantenimiento tanto de los caracoles de las diferentes procedencias como de las cepas de S. mansoni. Los ejemplares de $B$. tenagophila procedentes de 13 localidades no eliminaron cercarias durante el período de observación de 90 días; tampoco se encontraron formas evolutivas del esquistosoma cuando se los examinó por aplastamiento. Solamente caracoles originarios de Fuente Salto y Ayolas se consiguieron infectar con la cepa SJ2. Los caracoles de Ayolas eliminaron cercarias a los 36 días de la exposición a 50 miracidios; los de Fuente Salto, a los 30 días de ser expuestos a solo 10 miracidios.

El porcentaje de infección fue 2\% (4 de 163) en los especímenes de Ayolas y 18\% (8 de 45) en los de Fuente Salto. De los controles B. glabrata se infectaron 31\% (131 de 424) de los expuestos a la cepa BH2; de los controles B. tenagophila, 76\% (196 de 258) de los expuestos a la cepa SJ y 60\% (54 de 90) de los expuestos a la SJ2.

Se encontraron huevos de S. mansoni en las heces de los 12 ratones sobrevivientes, 5 infectados con las cercarias eliminadas por B. tenagophila de Ayolas y 7 de Fuente Salto. La perfusión de estos ratones mostró distribución y número medio de esquistosomas en el hígado y mesenterio comparables a los obtenidos en infestaciones experimentales de mantenimiento del ciclo de la cepa SJ2.

\section{DISCUSIÓN}

Desde hace aproximadamente siete décadas se conocen dos especies de caracoles planórbidos, B. glabrata y B. straminea, como hospederos intermediarios de $S$. mansoni en la región neotropical. La función de hospedero intermediario de la tercera especie, B. tenagophila, fue comprobado en investigaciones realizadas en 1956 en el estado brasileño de São Paulo (19).

La distribución geográfica de $B$. tenagophila abarca una extensa región de Sudamérica. Según Malek (20) este caracol se encuentra en Argentina, Bolivia, Paraguay, Perú y Uruguay. Sin embargo, solamente en el Brasil es actualmente endémica la esquistosomiasis y la especie $B$. tenagophila se ha comprobado transmisora natural en varios estados de este país. Ya en 1957 se describieron focos activos en Rio de Janeiro (21). En 1981 se notificaron casos autóctonos en Santa Catarina (22) y en 1985 se detectaron B. tenagophila naturalmente infectados en dos municipios del estado de Minas Gerais
(23, 24). En 1991 se detectaron casos en Jaraguá do Sul, Santa Catarina (25), lugar este el más cercano a la Argentina en el que se ha detectado esquistosomiasis. Los índices de infección natural de los caracoles transmisores encontrados en los focos mencionados oscilaron entre 0,1 y 17\%. El máximo índice de infección natural de B. tenagophila, 81\%, fue hallado en 1969 en Pedro Toledo, São Paulo (26).

La progresiva expansión de la esquistosomiasis hacia la región de influencia de la cuenca del río de la Plata, donde viven poblaciones de $B$. tenagophila, hizo que comenzara a investigarse la potencialidad de esta especie como transmisora. Los primeros ensayos de susceptibilidad se realizaron en 1968. Pellegrino et al. (4) expusieron sin éxito $B$. tenagophila de Puerto Hormiguero, localidad situada a orillas del río Uruguay (Santo Tomé, Corrientes, Argentina), a cepas de S. mansoni adaptadas a B. glabrata (LE) y B. tenagophila (SJ). En 1975, Chieffi (27) llamó la atención sobre la variación de la susceptibilidad de B. tenagophila a infección esquistosomiásica. Dicha susceptibilidad variaría desde 0\% hasta más de $80 \%$ dependiendo de la procedencia de los caracoles. Así los ejemplares de Londrina (Paraná, Brasil), a pesar de vivir en lugares donde existen casos de esquistosomiasis, son refractarios a tres cepas de $S$. mansoni, las SJC y GB adaptadas a B. tenagophila y la LDN adaptada a B. glabrata. En otra investigación (28), los caracoles de São José dos Campos mostraron una susceptibilidad de $81 \%$ a la cepa simpátrida (SJC) y una menor susceptibilidad a la cepa GB; los ejemplares de B. tenagophila de Diadema, São Paulo, mostraron hasta $30 \%$ de infección con la SJC y $19 \%$ con la GB.

En 1976 se intentó nuevamente infectar B. tenagophila de Puerto Hormiguero con $S$. mansoni, con resultados negativos, pese a la aplicación de rayos gamma (1000 a $7000 \mathrm{R})$ a los caracoles antes de su exposición individual y en masa a miracidios de las cepas LE y SJ (29). En 1978 se ensayó la susceptibilidad de $B$. tenagophila de 
varias regiones a la infección con la cepa SJ y se hallaron 15 poblaciones susceptibles entre las 20 estudiadas, con tasas de infestación de 1 a $91 \%$ (30). Entre las poblaciones que no se infectaron dos procedieron de la cuenca del río Uruguay, una de Paysandú (Uruguay) y la otra de Puerto Hormiguero, verificándose por tercera vez la refractariedad de $B$. tenagophila de esta última procedencia. Asimismo, en 1983 se demostró experimentalmente la variabilidad de la susceptibilidad de B. tenagophila en un área endémica, Belo Horizonte (Minas Gerais, Brasil) y zonas adyacentes (31). En esa investigación los ejemplares de Sabará fueron refractarios a las cepas LE - adaptada a B. glabrata-, SJ y SS esta última aislada de un roedor silvestre, Holochilus brasiliensis. Por el contrario, los de Belo Horizonte fueron susceptibles a las cepas LE (4\%) y SJ $(22 \%)$, en tanto que los de Nova Lima se infectaron con las cepas SJ (6\%) y SS (2\%). Más recientemente, en 1987, Paraense y Correa (32) alertaron sobre la probable propagación de la esquistosomiasis al sur del Brasil, en un área no endémica, en las cercanías de la cuenca del río Uruguay. Estos autores consiguieron infectar con la cepa SJ2 a $2 \%$ de especímenes de $B$. tenagophila originarios de Tramandaí, ciudad turística del litoral atlántico en el estado de Rio Grande do Sul.

En este trabajo, en que todas las poblaciones de B. tenagophila ensayadas procedían de áreas exentas de esquistosomiasis, se ha comprobado también que su susceptibilidad a S. mansoni es variable, con predominio de las poblaciones inmunes sobre las susceptibles. Entre las que no se infectaron estaban las de la provincia de Corrientes (Curuzú Cuatiá, Caá-Catí y San Roque) que se expusieron en masa a los miracidios (cuadro 1). Las experiencias de susceptibilidad se iniciaron con caracoles de esta procedencia, utilizando la exposición en masa, que tiene sobre la individual la ventaja de que los caracoles son expuestos en los acuarios a gran número de huevos de los que eclosionan los miracidios en diferentes momentos, con lo cual el índice de infección puede duplicarse (33). Los moluscos en los acuarios son sometidos así al ataque escalonado de varias poblaciones de miracidios, lo que haría aumentar la posibilidad de infección, tal como se comprobó con ejemplares de $B$. glabrata procedentes de Belo Horizonte, Minas Gerais (29). Esta especie resultó infectada usando la exposición en masa con la cepa PT adaptada a B. tenagophila de Pedro Toledo, São Paulo. La cepa había sido aislada de 15 caracoles de esa especie naturalmente infectados. Sin embargo, la especie $B$. glabrata resultó refractaria en otros ensayos (16) cuando se expusieron especímenes individualmente a la cepa SJ.

No obstante estos antecedentes, en el presente estudio no se utilizó la exposición en masa con todas las muestras de $B$. tenagophila, sino que se prefirió la exposición individual por ser sus resultados más comparables.

Las poblaciones que resultaron susceptibles se infectaron con la cepa SJ2, pero en bajas proporciones, de 2 a $18 \%$. Las cercarias de la cepa SJ2 eliminadas por B. tenagophila de Ayolas y Fuente Salto evolucionaron en los ratones hasta gusanos adultos normales que pusieron huevos viables.

Es necesario señalar (cuadro 1) que varias poblaciones de $B$. tenagophila que no se infectaron fueron expuestas solamente a los miracidios de la cepa $\mathrm{BH} 2$, adaptada a $B$. glabrata. La probabilidad de infección es mayor cuando se utiliza la cepa de esquistosoma adaptada a la misma especie. En consecuencia, aún tenemos que verificar la susceptibilidad de esas poblaciones a la cepa SJ2. La susceptibilidad a $S$. mansoni de B. tenagophila, que vive en aguas superficiales de las cuencas del Paraná y del Uruguay, tiene importancia epidemiológica.

Ayolas es una pequeña localidad paraguaya en expansión, ubicada sobre la margen derecha del Paraná, donde está emplazada la represa de Yacyretá. El puente internacional construido con la represa une la citada localidad con la de Ituzaingó (Corrien- tes, Argentina) y por él circula un tránsito importante.

El sistema de vigilancia epidemiológica que funcionó en el área de la represa entre 1975 y 1989 probablemente contribuyó a impedir la propagación de la esquistosomiasis a esta región. Entre noviembre de 1980 y mayo de 1981 se encontraron huevos de $S$. mansoni en las heces de cuatro trabajadores. Estos participaban en la construcción del puente sobre el brazo Añá-Cuá afluente del río Paraná, que une territorio paraguayo con la isla Yacyretá (observaciones inéditas de los autores). Los obreros eran brasileños y provenían de áreas endémicas. Fueron tratados con oxamniquina y mantenidos bajo control hasta que no se hallaron huevos de esquistosoma en sus heces. No obstante, ante el riesgo de que pudieran haber contaminado con sus excrementos cursos de aguas en los que existen criaderos de B. tenagophila se efectuó un sondeo epidemiológico, tanto en los caracoles como en los obreros que compartían el trabajo y frecuentaban los mismos lugares que las personas en las que se detectó esquistosoma. Los resultados fueron negativos.

Se debe señalar que con las aguas del embalse de Yacyretá está previsto construir canales de riego agrícola, tanto en territorio argentino como paraguayo.

Fuente Salto está situada en las cercanías de un polo de desarrollo creado en torno de la represa binacional de Santo Grande, construida en la cuenca del río Uruguay durante los años setenta como proyecto conjunto de la Argentina y el Uruguay. En la parte superior de la represa un puente facilita el tránsito entre la ciudad uruguaya de Salto y la argentina de Concordia.

Teniendo en cuenta la amplia distribución geográfica de $B$. tenagophila en la cuenca de los ríos Paraná y Uruguay y el problema no resuelto de las migraciones sin control sanitario, es necesaria la vigilancia epidemiológica permanente con el objetivo de detectar la posible aparición de focos de esquistosomiasis en la región. Sobre todo, cuando se han demostrado en otras 
áreas de Sudamérica nuevos focos de la enfermedad transmitida por este caracol planórbido. Periódicamente deberán hacerse encuestas epidemiológicas, que incluyan también la recolección y examen de caracoles, principalmente poblaciones de B. tenagophila, que es el que presenta en esta zona mayor potencial como hospedero intermediario de S. mansoni.

Agradecimiento. Los autores expresan su agradecimiento a Wladimir Lobato Paraense y a Lygia Correa, del
Departamento de Malacología del Instituto Oswaldo Cruz (Rio de Janeiro), que facilitaron las cepas de $S$. mansoni para la investigación; y a Luis Armando Mosqueda y Osvaldo David Benítez, del Centro Nacional de Parasitología y Enfermedades Tropicales (Corrientes, Argentina).

\section{REFERENCIAS}

1. Utroska JA, Chan MG, Dixon E, Yoon et al. An estimate of global needs for praziquantel within schistosomiasis control programmes. Geneva: World Health Organization, Division of Control of Tropical Diseases; 1989:1-92. (Documento de distribución reservada).

2. Paraense WL. Distribução dos caramujos no Brasil. En: Reis FA, Faria II, Katz N. Modernos conhecimentos sobre esquistossomose mansônica, Belo Horizonte. Academia Mineira de Medicina (Suplemento, Anais da Academia Mineira de Medicina, 1983-1984) 1986;1:117-128.

3. Romaña C, Silva AO, Romaña MS. Acerca de una investigación sobre esquistosomiasis realizada en la frontera argentino-brasileña de Misiones. An Inst Med Reg (Chaco, Argentina) 1957;4:265-269.

4. Pellegrino J, Borda CE, Valetti JA, Dibetto J. Observações sobre provas imuno-serológicas para esquistossomose mansoni realizadas em habitantes das provincias de Corrientes y Misiones, Argentina. Rev Inst Med Trop São Paulo 1968;10:277-286.

5. Borda CE. La represa de Itaipú y la probable propagación de la esquistosomiasis del Brasil a las provincias de Misiones y Corrientes. Resistencia, Chaco, Argentina: Universidad Nacional del Nordeste; 1975:1-15.

6. Borda CE, Rea MJF, Dhó MC. Vigilancia e investigación epidemiológica de las enfermedades parasitarias de la zona de YacyretáApipé (Paraguay-Argentina), Ayolas, Santiago e isla Yacyretá. En: Seminario sobre Medio Ambiente y Represas, Universidad de la República, Facultad de Humanidades y Ciencias, Montevideo, Uruguay, 2-14 de mayo de 1977. Montevideo: Organización de Estados Americanos, Dpto. Asuntos Científicos y Tecnológicos; Tomo 2, 1977:32-42.

7. Borda CE, Dhó MC, Rea MJF. Investigaciones epidemiológicas sobre la existencia de esquistosomiasis de Manson en las islas Apipé Chico y Apipé Grande. Rev Fac Med Univ Nac Nordeste (Corrientes, Argentina) 1978;1:6-17.

8. Borda CE, Rea MJF. Distribución geográfica de Biomphalaria potencialmente transmisoras de la esquistosomiasis en las cuencas de los ríos Paraná y Uruguay. En: Programa e Resumos, II Congresso Latinoamericano de Malacologia, 11-16 de julho de 1995, Pontificia Universidade Católica do Rio Grande do Sul. Comitê Permanente de Congressos Latinoamericanos de Malacologia, Sociedade Brasileira de Malacologia. Porto Alegre: Epecé Gráfica; 1:126.
9. Deslandes N. Técnica de dissecação e exame de planorbideos. Rev Serv Esp Saude Publica (Rio de Janeiro) 1951;4:371-382.

10. Paraense WL. The nomenclature of Brasilian planorbids, II-Australorbis tenagophilus (Orbigny, 1835). Rev Brasil Biol (Rio de Janeiro) 1961;21:143-349.

11. Paraense WL, Correa L. Susceptibility of Biomphalaria amazonica to infection with the strains of Schistosoma mansoni. Rev Inst Med Trop São Paulo 1971;13:387-390.

12. Paraense WL, Correa LA. A potential vector of Schistosoma mansoni in Uruguay. Mem Inst Oswaldo Cruz 1989;84(3):281-288.

13. Files VS. A study of the vector-parasite relationships in Schistosoma mansoni. Parasitology 1951;264-269.

14. Standen OD. Experimental infection of Australorbis glabratus with Schistosoma mansoni: I-Individual and mass infection of snail: the relationship of infection temperature and season. Ann Trop Med Parasitol 1952;46:48-53.

15. Chaia G. Técnica para concentração de miracidios. Rev Brasil Malariol Doenças Trop 1956;8:355-357.

16. Paraense WL, Correa LR. Variation in susceptibility of populations of Australorbis glabratus to strain of Schistosoma mansoni. Rev Inst Med Trop São Paulo 1963;5:15-22.

17. Borda CE, Pellegrino J. An improved thicksmear for quantitative diagnosis of Schistosoma mansoni infection. Rev Inst Med Trop São Paulo 1971;10:71-75.

18. Pellegrino J, Siqueira AF. Técnica de perfusão para colheita de Schistosoma mansoni em cobaios experimentalmente infestados. Rev Brasil Malariol Doenças Trop 1956;8:589-597.

19. Paraense WL, Deslandes N. Australorbis nigricans as the transmissor of schistosomiasis in Santos, state of São Paulo. Rev Brasil Malariol Doenças Trop 1956;8:235-245.

20. Malek EA. Snail hosts of schistosomiasis and other snail-transmitted diseases in tropical América: a manual. Washington, DC: Pan American Health Organization; 1985. (Scientific Publication 478).

21. Martins RS. Focos ativos de esquistossomose em Niteroi, estado de Rio de Janeiro. Rev Brasil Malariol Doenças Trop 1957;9:361-364.

22. Bernardini OJ, Machado MM. Isolamento de Schistosoma mansoni do primero foco de transmissão activa em São Francisco do Sul. Arq Med Catarinenses (Santa Catarina) 1981;10:213.

23. Melo AL de, Pereira LH. On the finding of Biomphalaria tenagophila naturally infected with
Schistosoma mansoni in the state of Minas Gerais, Brasil. Rev Inst Med Trop São Paulo 1985;27:99-101.

24. Carvalho OS, Souza CP, Katz N. Primeiro encontro de Biomphalaria tenagophila (Orbigny, 1935) naturalmente infectada con Schistosoma mansoni em Itajubá, sul do estado de Minas Gerais, Brasil. Rev Saude Publica 1985;19:88-91.

25. Espínola KS, Machado MM, Hoffmann PRP. Natural and experimental infections of planorbids from the island of Santa Catarina (Brasil). Rev Inst Med Trop São Paulo 1992; 34:289-294.

26. Piza JT, Ramos AS, Costa FA, Puppi AM, Barros NA. A esquistossomose no estado de São Paulo. Arq Hig Saude Publica (São Paulo) 1969;34:5-23.

27. Chieffi PP. Susceptibilidade e infecção por Schistosoma mansoni de cepas de Biomphalaria tenagophila originarias dos estados de São Paulo e Paraná. Rev Inst Med Trop São Paulo 1968;17:92-96.

28. Paraense WL, Correa L. Susceptibility of Australorbis tenagophilus to infections with Schistosoma mansoni. Rev Inst Med Trop São Paulo 1963b;5:23-29.

29. Borda CE, Pellegrino J. Susceptibilidad de Biomphalaria tenagophila y B. glabrata a dos cepas de Schistosoma mansoni. Rev Inst Med Trop São Paulo 1976;18:157-164.

30. Paraense WL, Correa L. Differential susceptibility of Biomphalaria tenagophila population to infection with a strain of Schistosoma mansoni. J Parasitol 1978;64:822-826.

31. Souza CP de, Araujo N, Madeira NG, Carvalho $\mathrm{O}$ dos S. Susceptibilidade de Biomphalaria tenagophila de Belo Horizonte e adyacencias a infecção com tres cepas de Schistosoma mansoni. Rev Inst Med Trop São Paulo 1983;25:168-172.

32. Paraense WL, Correa L. Probable extension of Schistosoma mansoni to southernmost Brazil. Mem Inst Oswaldo Cruz 1987,82 4):577.

33. Moore D. Efficacy of mass exposure of Australorbis glabratus to Schistosoma mansoni. J Parasitol 1964:50:798-799.

Manuscrito recibido el 30 de noviembre de 1995 y aceptado para publicación en versión revisada el 20 de agosto de 1996. 
ABSTRACT To study the possibility that schistosomiasis might be able to spread into the basins of the Paraná and Uruguay rivers, 1711 snails were experimentally exposed to infection with Schistosoma mansoni. These snails were laboratory-bred progeny of Biomphalaria tenagophila collected from 15 populations in the geographic area under consideration. Three strains of $S$. mansoni were used: $\mathrm{BH} 2$, adapted to B. glabrata, and SJ and SJ2, adapted to $B$. tenagophila. None of the 543 snails exposed to the $\mathrm{BH} 2$ strain became infected, nor did any of the 668 exposed to SJ. However, of the 500 exposed the SJ2, 4 of 163 snails (2\%) from Ayolas, a locality in the Paraná basin, were susceptible, as were 8 of $45(18 \%)$ from Fuente Salto, in the Uruguay basin. These findings are similar to those of studies done in other geographic areas, where some populations of B. tenagophila do not become infected with S. mansoni while others are susceptible. The results of this study signal the possibility of the expansion of schistosomiasis into a broad region of South America in which B. tenagophila is found.

$4^{\circ}$ Simposio Internacional: Administración de la Higiene y la Salud en el Medio Laboral $\begin{array}{ll}\text { Nuevas fechas: } & 27 \text { a } 29 \text { de abril de } 1998 \\ \text { Lugar: } & \text { Oostende, Bélgica }\end{array}$

Se avisa a los interesados que este simposio ya no se celebrará del 13 al 15 de octubre de 1997 como se había planeado originalmente. Las nuevas fechas, del 27 al 29 de abril de 1998, son definitivas.

Los resúmenes de trabajos propuestos para presentación deberán llegar a manos de la secretaría el 1 de abril de 1997 a más tardar. Solo se aceptarán trabajos originales que describan formas innovadoras de enfocar asuntos clave. El comité científico hará su selección sobre la base de los resúmenes. Los autores recibirán la decisión final e instrucciones para la preparación de manuscritos hacia fines de mayo de 1997. La versión final de los trabajos debe estar lista para el 1 de diciembre de 1997.

Información:

4th International Symposium: Hygiene and Health Management

in the Working Environment

c/0 TI vzw, attn. Rita Peys

Desguinlei 214

B - 2018-Antwerpen

Teléfono: +32 (3) 2160996

Fax: +32 (3) 2160689

Correo electrónico: HYGIENE@TI.KVIV.BE 